\title{
Performance assessment of a homodyne laser radar for surface displacement monitoring
}

\author{
Alejandro Rodriguez \\ Adolfo Comeron, MEMBER SPIE \\ Eloy Mesalles \\ Universitat Politècnica de Catalunya \\ (UPC) \\ Departament de Teoria del Senyal i \\ Comunicacions \\ Electromagnetics and Photonics \\ Engineering Group \\ Campus Nord UPC \\ Edifici D-4, C/Jordi Girona, 1-3 \\ E-08034 Barcelona, Spain \\ E-mail: alejandro@tsc.upc.es
}

\begin{abstract}
Measurements under varied conditions performed with a homodyne laser radar prototype for surface displacement monitoring are presented. They include constant and variable velocity measurements from both a disk and a belt target. In this manner, a methodology for estimating the probability of detection and the standard deviation of the complete system is proposed. (๑) 2001 Society of Photo-Optical Instrumentation Engineers. [DOI: 10.1117/1.1419189]
\end{abstract}

Subject terms: laser radar; homodyne; optical mixing; velocity measurement; surface displacement measurement; HeNe laser; avalanche photodiode; Doppler shift; coherence; signal acquisition.

Paper 200467 received Nov. 27, 2000; revised manuscript received May 1, 2001; accepted for publication July 6, 2001.

\section{Introduction}

Some homodyne laser radar prototypes have been proposed in previous literature. For instance Rudd ${ }^{1}$ and Churnside ${ }^{2,3}$ presented prototypes with optical mixing taking place inside the laser oscillator. Liskow et al. ${ }^{4}$ presented a very interesting discussion on the effect on the probability of detection and probability of false alarm of the transmitted laser beam width. More recently, Gatt et al. ${ }^{5}$ theoretically and experimentally presented the influence of the different noise sources in the velocity measurement precision.

We have reported ${ }^{6}$ a prototype based on a $\mathrm{HeNe}$ laser with optical mixing taking place on the surface of an external avalanche photodiode (APD). This enables performing optical homodyning between signals whose frequencies differ in more than $300 \mathrm{MHz}$, while the prototypes based on a $\mathrm{HeNe}$ laser that use the laser as a mixer cannot perform mixing between signals whose frequency differ more than $\sim 100 \mathrm{kHz}$ (Ref. 7).

In this paper, we present the performance assessment of the system presented in Ref. 6. In Sec. 2 some considerations of signal-frequency counting are discussed; they conclude that the frequency cannot be directly measured if the SNR is not high enough, which is our case. Section 3 describes the subsystem used to count the frequency of the received signal, based on the spectrum analyzer principle. In Sec. 4 the methodology for performance assessment is defined, and finally, Sec. 5 describes the experimental results. More detailed results can be found in Ref. 8.

\section{Noise Effects in Frequency Estimation}

In a coherent laser radar system, many noise mechanisms must be born in mind (see, for example, Ref. 5). In the following we consider that, as a result, the electrical output signal is affected by an additive Gaussian white noise (AGWN).

Many authors have modeled the process of frequency counting in an AGWN environment. For instance, Papoulis ${ }^{9}$ describes the frequency counting as a zero- crossing count and computes its rate $\lambda_{0}$ (twice the frequency) for a signal $x(t)$, as

$\lambda_{0}=\frac{1}{\pi}\left[\frac{\int_{-\infty}^{\infty} \omega^{2} S_{x}(\omega) \mathrm{d} \omega}{\int_{-\infty}^{\infty} S_{x}(\omega) \mathrm{d} \omega}\right]^{1 / 2}$

where $S_{x}(\omega)$ is the power spectral density of $x(t)$.

For a normalized sinusoidal signal plus a white Gaussian additive noise $n(t)$ :

$x(t)=\sin \left(\omega_{0} t\right)+n(t)$,

where $S_{x}(\omega)$ can be written as

$$
\begin{aligned}
S_{x}(\omega)= & \frac{1}{4}\left[\delta\left(\omega-\omega_{0}\right)+\delta\left(\omega+\omega_{0}\right)\right] \\
& + \begin{cases}\frac{N_{0}}{2} & \text { for }|\omega|<\mathrm{BW} \\
0 & \text { for }|\omega|>\mathrm{BW},\end{cases}
\end{aligned}
$$

where BW is the measuring system bandwidth, considered as an ideal filter, and $N_{0}$ is the bilateral noise power spectral density. Note that $N_{0}$ can be related to the SNR at the input of the system, considering $x(t)$ normalized:

$N_{0}=\frac{1}{2 \text { SNR BW }}$.

We can now compute the rate $\lambda_{0}$ : 


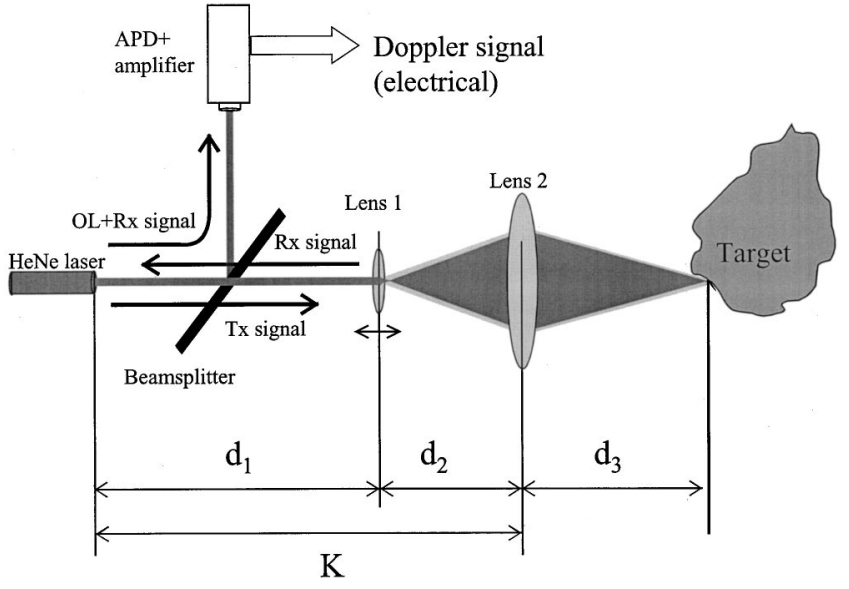

Fig. 1 Prototype optics layout (Ref. 6).

$$
\begin{aligned}
\lambda_{0} & =\frac{1}{\pi}\left[\frac{\left(\mathrm{BW}^{3} / 3\right) N_{0}+\omega_{0}^{2} / 2}{\mathrm{BW} N_{0}+1 / 2}\right]^{1 / 2} \\
& =\frac{1}{\pi}\left[\frac{\mathrm{BW}^{2} /(6 \mathrm{SNR})+\omega_{0}^{2} / 2}{1 /(2 \mathrm{SNR})+1 / 2}\right]^{1 / 2}= \begin{cases}2 f_{0} & \text { for } \mathrm{SNR} \gg \\
\frac{\mathrm{BW}}{\sqrt{3} \pi} & \text { for } \mathrm{SNR} \ll .\end{cases}
\end{aligned}
$$

According to Eq. (5), when the SNR takes a high value, the frequency count is correctly made; for low SNR values, the obtained count does not correspond to the desired value but to the system bandwidth.

Reference 6 includes preliminary measurements obtained with a homodyne laser radar system that can be seen in Fig. 1. The signal and noise power measures can be seen in Figs. 2(a) and 2(b). These measurements have been taken using a spectrum analyser using a resolution bandwidth (RBW) equal to $100 \mathrm{kHz}$. The maximum signal power measured is $-30 \mathrm{dBm}$, overcoming a noise floor spectral power density $N_{0}=-50 \mathrm{dBm} /(100 \mathrm{kHz})$. This means that, considering a total system bandwidth $\mathrm{BW}=100 \mathrm{MHz}$, our system presents an $\mathrm{SNR}=-10 \mathrm{~dB}$, not allowing for straight frequency counting.

The use of spectrum analysis techniques makes the frequency estimation of signals in a noisy environment easier by reducing the system input bandwidth. The measures presented in this paper are made using these techniques.

\section{Signal Conditioning and Acquisition and Frequency Counter Subsystem}

The laboratory setup includes a specific subsystem that performs the signal conditioning and acquisition and also the frequency counting. Figure 3 shows the general scheme of this subsystem. It will be denominated by carrier seeker in the following.

First, the Doppler signal coming out of the photoreceiver of the laser radar system is introduced in an ad hoc spectrum analyzer module. We use this module to extract the Doppler signal spectral peak from the noisy output of the

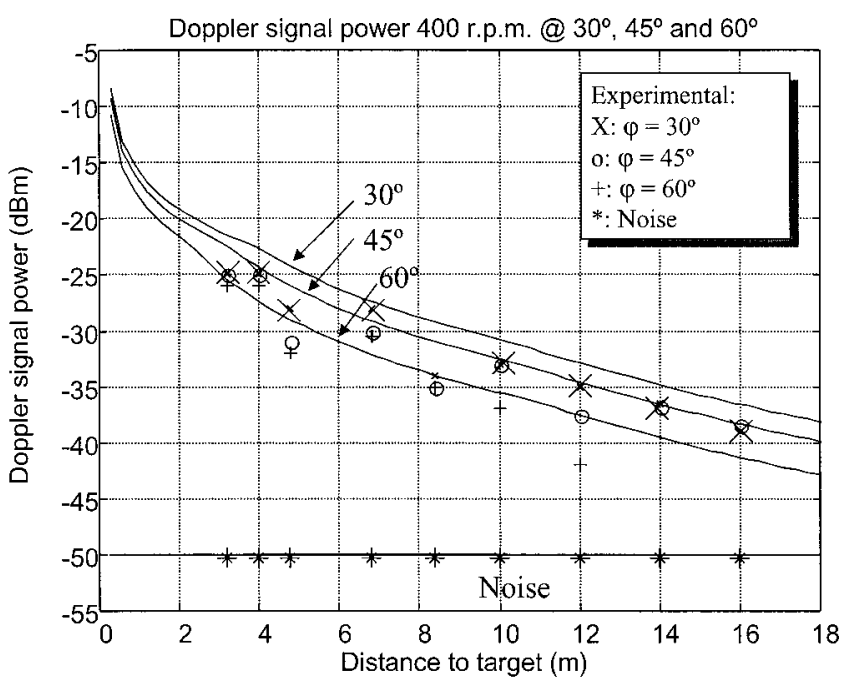

(a)

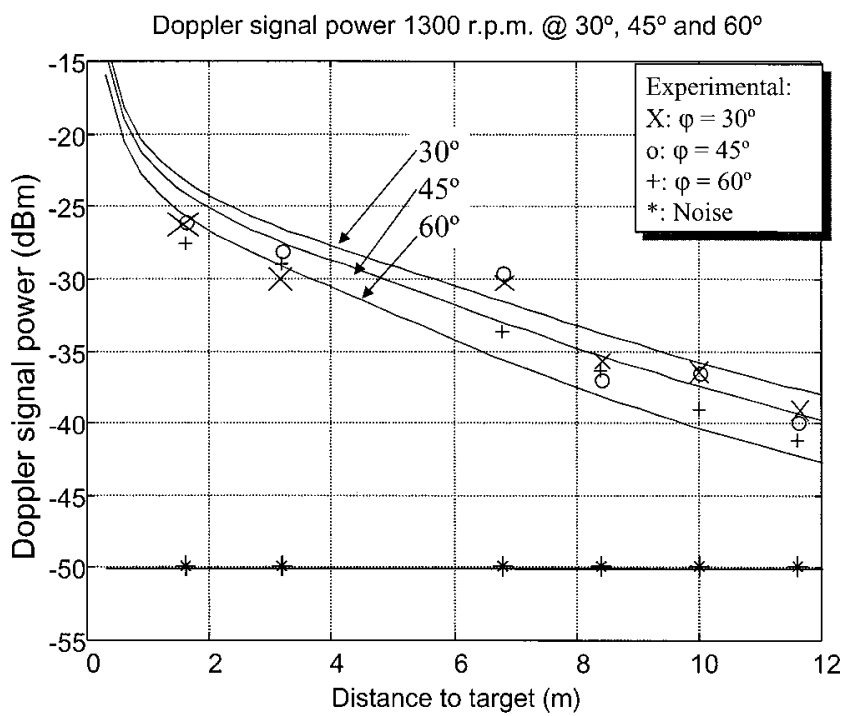

(b)

Fig. 2 Doppler signal power for the target rotating at (a) $400 \mathrm{rpm}$ and (b) $1300 \mathrm{rpm}$ (Ref. 6).

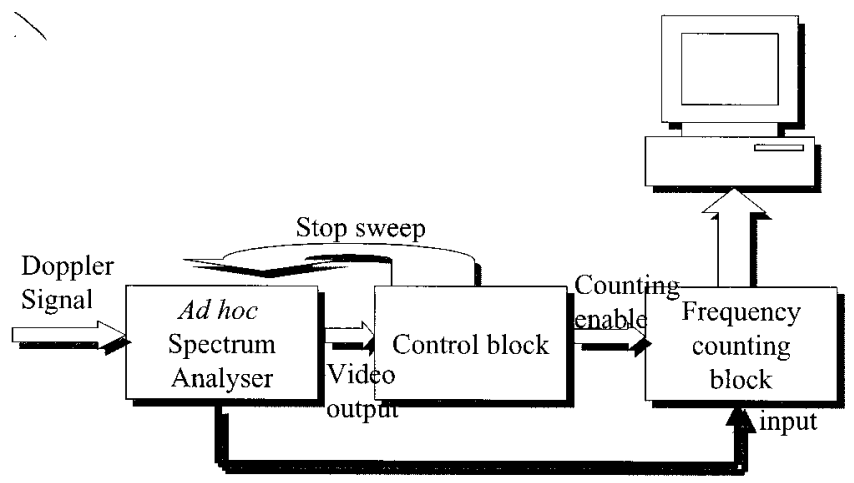

First LO

Fig. 3 General scheme of the signal conditioning and acquisition and frequency counter subsystem (carrier seeker). 
photoreceiver. Our past experience measuring with a general purpose spectrum analyzer suggested the following operating parameters:

1. intermediate frequency (IF): $150 \mathrm{MHz}$

2. RBW: $600 \mathrm{kHz}$

3. first local oscillator sweeping range: 160 to $250 \mathrm{MHz}$

4. second local oscillator frequency:150 MHz

The subsystem includes a frequency-counting unit whose main characteristics are

1. prescaler: 10

2. crystal reference frequency: $2.018 \mathrm{MHz}$

3. counting interval: $507.4 \mu$ s (1024 reference clock pulses)

4. number of bits: 14

5. maximum measurable frequency: $320 \mathrm{MHz}$

According to the previous figures, the ad hoc spectrum analyzer can detect signals whose frequency is in the band between 10 and $100 \mathrm{MHz}$. When a signal peak is detected at its output, the sweeping stops. The control block then orders the frequency counting block to begin counting the frequency of a sample taken from the first local oscillator, located between 160 and $250 \mathrm{MHz}$ : thus, the frequency counting block has at its input a signal with a good SNR value and whose frequency is related, in a known way to the Doppler signal frequency.

The frequency counts obtained by the carrier seeker from a target in a certain observation interval are called elementary counts and they must be preprocessed. We have observed that the elementary counts obtained when the first local oscillator sweeps in an ascendant way are slightly smaller than the real signal frequency, while counts obtained in descendent sweepings are slightly greater than the correct value. This biasing can be appreciated in Fig. 4, where the elementary counts obtained in a certain observation interval are presented in a histogram. To avoid this problem, the preprocessing software averages the counts obtained during the observation interval in ascending and descending sweeps separately, calculating afterward the semisum of both values: the result will be called an individual count in the rest of the paper. If the subsystem has not been able to obtain all the components to calculate an individual count, a fail is written down.

The successive individual count values are presented to the system operator; statistical calculations based on these data are also presented.

Before every measuring session, the carrier seeker was thoroughly calibrated with the aid of a microwave frequency synthesizer.

\section{Methodology for Performance Assessment}

Two main figures were used to assess the performance of our system: the probability of detection estimate $P_{D}$ and the standard deviation in velocity estimation $s$. In this section, both are defined.

Liskow et al. ${ }^{4}$ presented a rigorous theoretical definition of probability of detection. In this section, a somewhat different definition is used, adapted to a system in which the

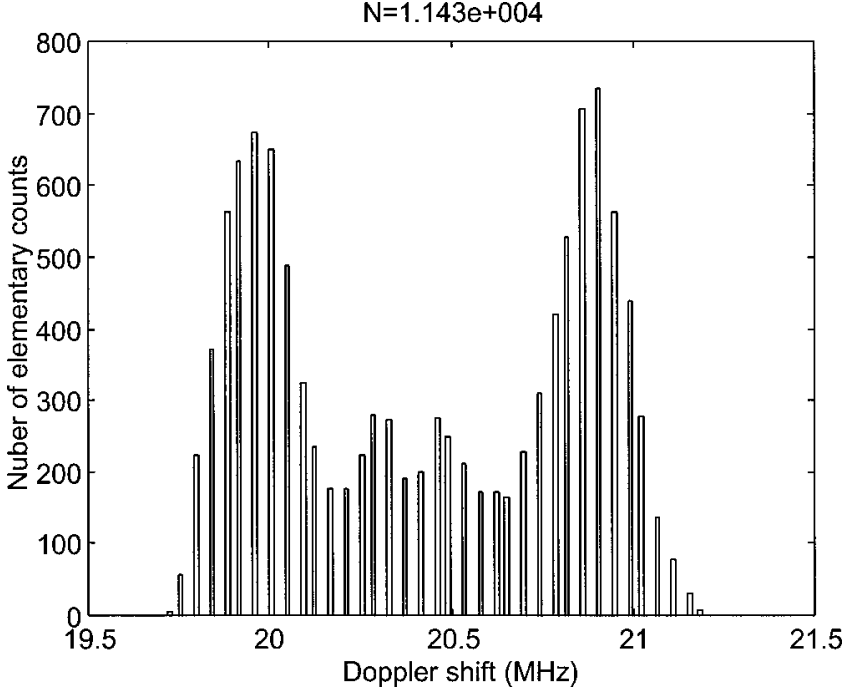

Fig. 4 Histogram of elementary counts with the target rotating at constant speed.

target is always illuminated by the laser transmitter. No $a$ priori calculations are made and all the results come from experimental data.

Gatt et al. ${ }^{5}$ presented both theoretical and practical considerations of the dependence on the SNR of the precision in velocity estimate, applied to different short and long range vibrometers that measure Doppler frequencies that do not exceed $250 \mathrm{~Hz}$.

In our experiment, Doppler shifts produced by the detected target speed are in the range between 20 and 70 $\mathrm{MHz}$, corresponding to velocities between 6 to $22 \mathrm{~m} / \mathrm{s}$. For detecting such Doppler shifts, the mixing cannot take place inside the laser resonator (see Refs. 1 and 7), so an external photodetector device (as shown in Fig. 1) must be used.

We tested the system behavior for four different counting intervals: 50, 100, and $500 \mathrm{~ms}$ and $1 \mathrm{~s}$. A statistically relevant number of individual count attempts $N$ were performed, so that mean and standard deviation values could be calculated. Individual counts that differ more than 2 $\mathrm{MHz}$ from the statistical median were discarded as out of range and considered as fails, so they are not presented in (a)

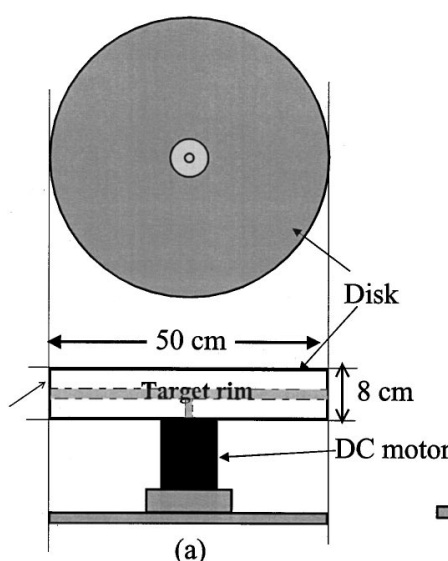

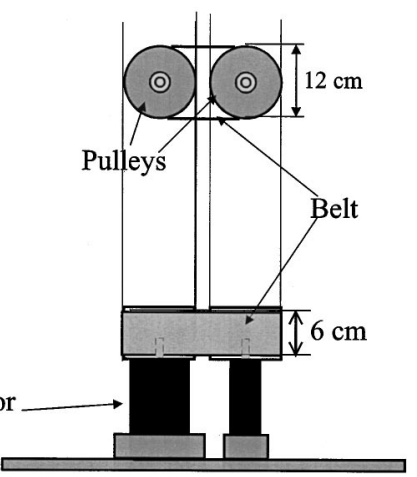

(b)
Fig. 5 Laboratory targets: (a) disk target and (b) belt target. 
Rodriguez, Comeron, and Mesalles: Performance assessment ...
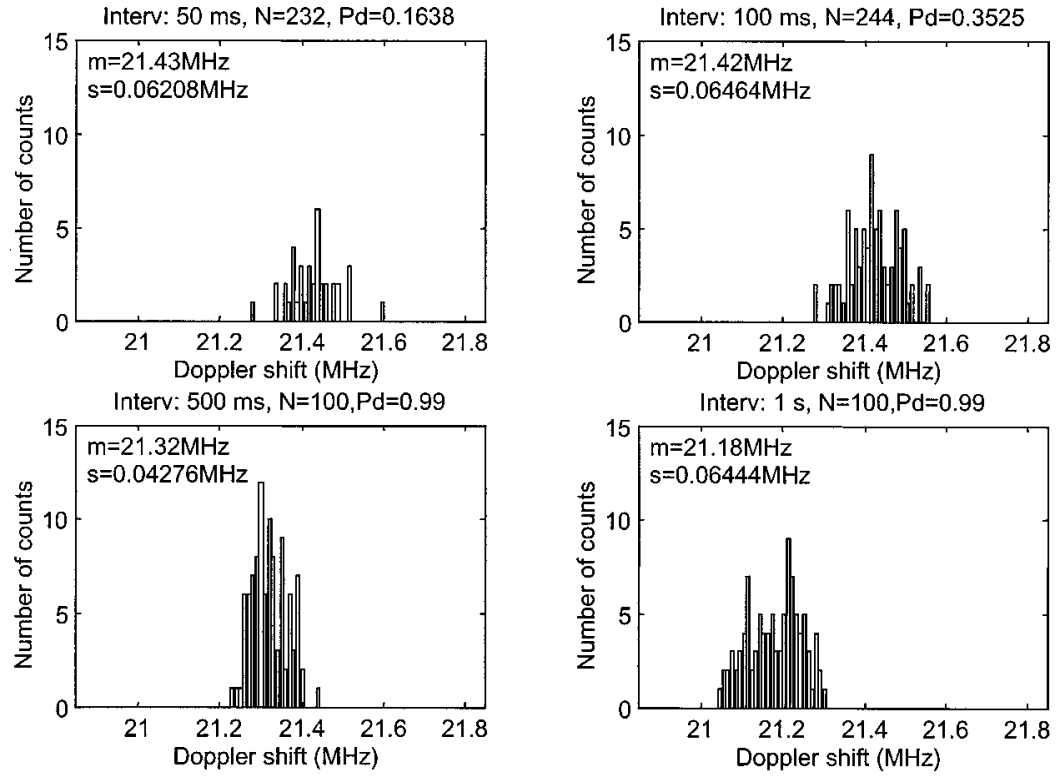

Fig. 6 Histograms of individual counts with the disk target at nominal distance $6 \mathrm{~m}$, an incidence angle of $30 \mathrm{deg}$, and rotating at $444 \mathrm{rpm}$.

the histograms. We defined the probability of detection estimate $P_{D}$ as the ratio of accepted individual counts divided by the total number of attempts $(N)$.

In Ref. 6 a criterion of tolerance in distance was defined. It took into account the possibility that the target was located at a distance different from the nominal one. This would imply that the transmitted laser beam does not have its waist on the target surface. Departures from the nominal distance provoke losses in the detected Doppler signal and thus, a performance worsening that is expected to diminish $P_{D}$. We performed measurements with the target located at the nominal position and displaced a certain distance, equal to the calculated tolerance, and we evaluated the corresponding loss in performance figures.

The standard deviation in frequency estimation $s$ is calculated as the standard deviation of the accepted counts. It can be related to the standard deviation in velocity estimation by the Doppler effect formula.

The accuracy of the experimental data was checked with an alternative system.

\section{Experimental Results}

Measurements were made on two different targets: a disk target and a belt target. The disk target was already de-
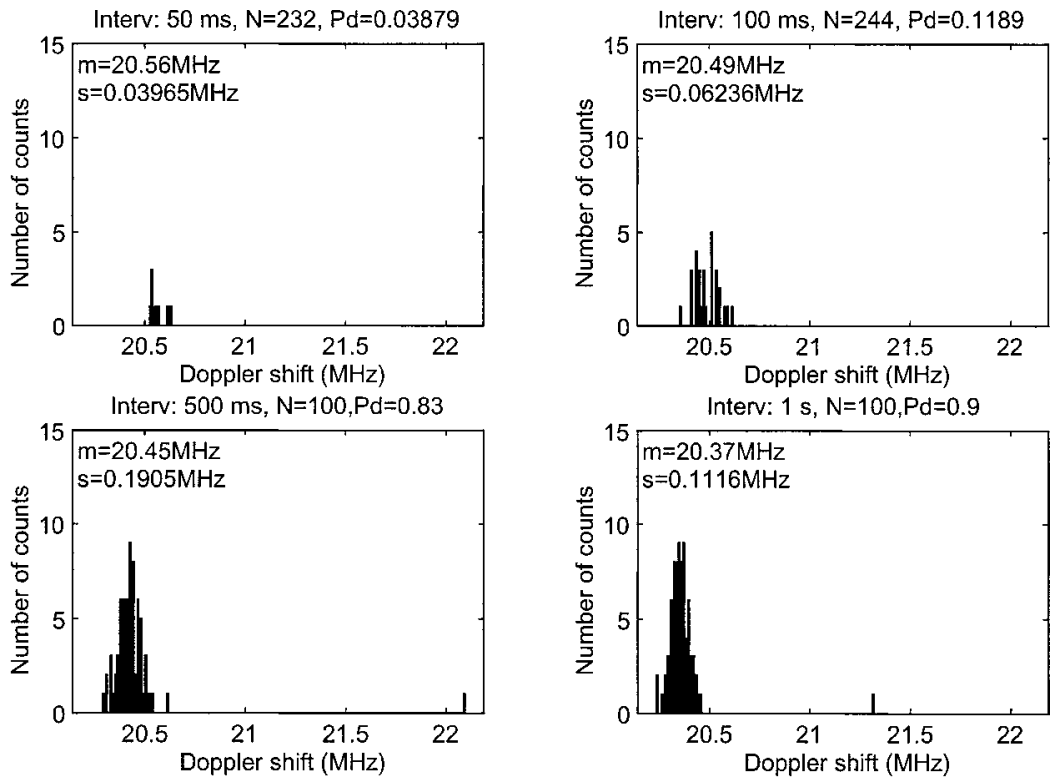

Fig. 7 Histograms of individual counts with the disk target at nominal distance $6 \mathrm{~m}$ plus $80 \mathrm{~cm}$ of tolerance displacement, incidence angle of $30 \mathrm{deg}$, and rotating at $444 \mathrm{rpm}$. 
Rodriguez, Comeron, and Mesalles: Performance assessment ...
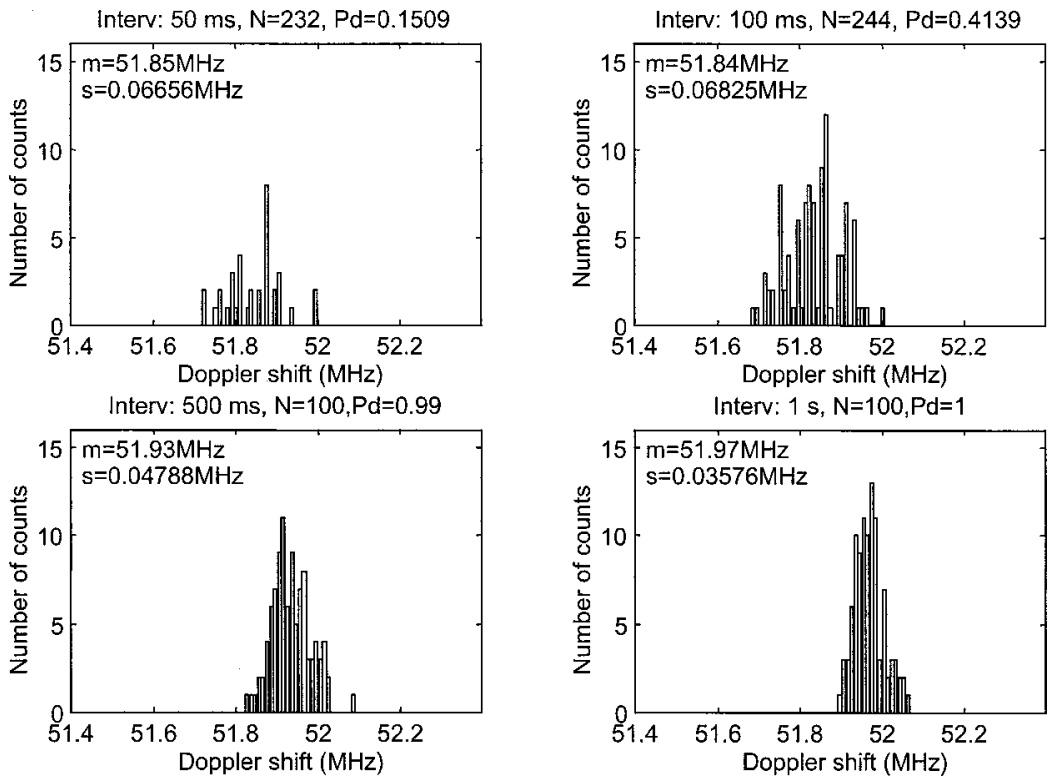

Fig. 8 Histograms of individual counts with the disk target at nominal distance $6 \mathrm{~m}$, incidence angle of $30 \mathrm{deg}$, and rotating at constant speed $1260 \mathrm{rpm}$.

scribed in Ref. 6. It is a 25 -cm-radius disk whose cylindrical surface is covered with paper, material with a high scattering value. Its maximum rotating speed is approximately $1300 \mathrm{rpm}$. The disk target includes a module that performs angular speed measurements.

The procedure to determine the incidence angle on the target surface shows a random error as high as $\pm 7 \mathrm{deg}$, so the accuracy of the measures is affected. In some of the measurements presented, this error is indicated.

The belt target consists in two parallel 6-cm-radius pulleys and a continuous belt whose surface displacement will be monitored. The pulleys rotate at a maximum speed of $\sim 1800 \mathrm{rpm}$. Both targets are represented in Fig. 5. No sys- tem is available to monitor the angular speed of the pulleys, so the indicated values have been calculated from the nominal data of the electric motor.

\subsection{Measurements with a Disk Target}

Although the measures with the disk target were performed at distances up to $7 \mathrm{~ms}$, at this distance the tolerance is severely reduced. Thus, the measurements presented here were taken at a maximum distance of $6 \mathrm{~m}$ for measures at constant speed. The measures at variable speed were taken at a 3-m distance.
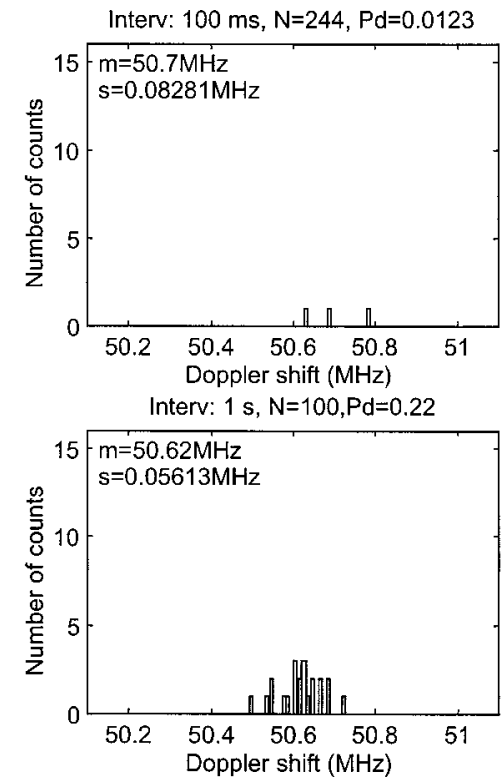

Fig. 9 Histograms of individual counts with the disk target at nominal distance $6 \mathrm{~m}$ plus $80 \mathrm{~cm}$ of tolerance displacement, incidence angle of $30 \mathrm{deg}$, and rotating at constant speed $1260 \mathrm{rpm}$.

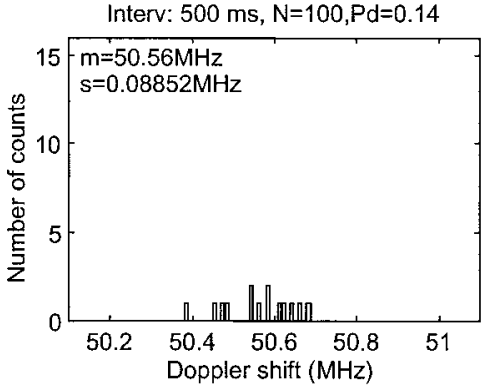


Rodriguez, Comeron, and Mesalles: Performance assessment ...
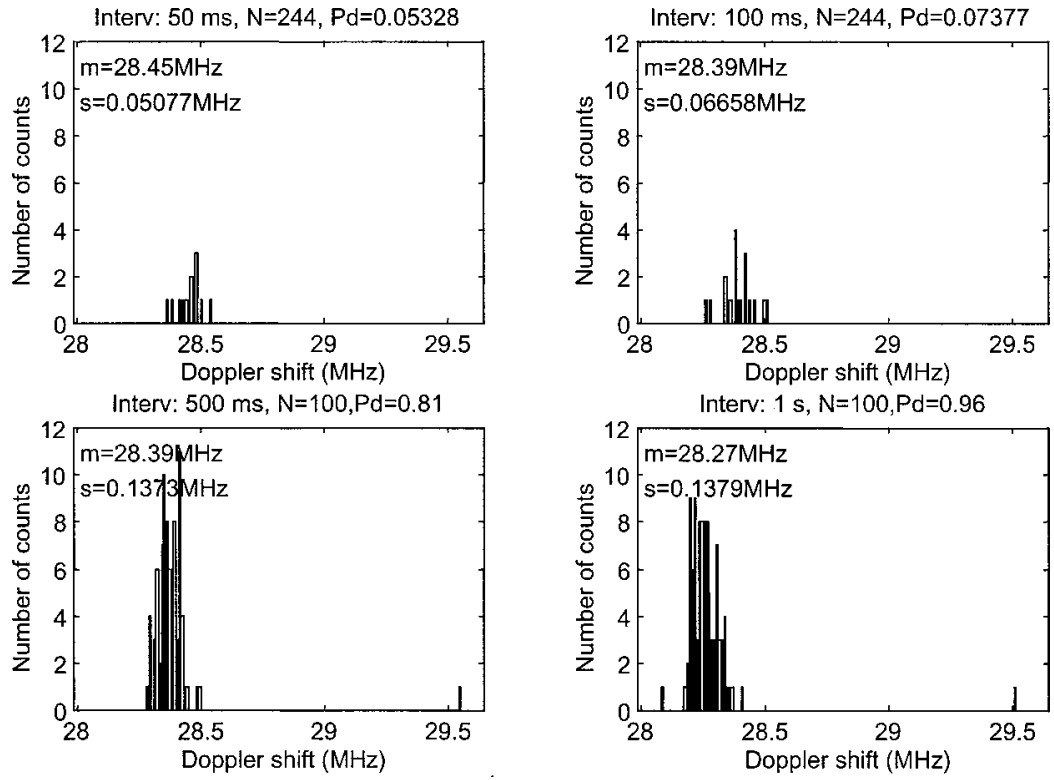

Fig. 10 Histograms of individual counts with the disk target at nominal distance $6 \mathrm{~m}$, incidence angle of $45 \mathrm{deg}$, and rotating at constant speed $444 \mathrm{rpm}$.

\subsubsection{Measurements at constant speed}

The first series of measurements were taken with the disk rotating at approximately $444 \mathrm{rpm}$, corresponding to a linear speed of the target rim of $11.6 \mathrm{~m} / \mathrm{s}$. Figure 6 presents the measurements taken with the target at the nominal position with an incidence angle $\varphi=30 \mathrm{deg}$, measured from the normal to the rim surface at the incidence point, for four different values of the observation interval: 50, 100, and $500 \mathrm{~ms}$, and $1 \mathrm{~s}$. The four-histogram series shows the increment of the estimated probability of detection with the observation interval. Also, the mean (marked as $m$ ) of the detected counts reduces slightly when the interval grows; we attribute this effect to the reduction in the relative weight of elemental measurements out of range. The standard deviation of the frequency estimate (marked in the histograms as $s$ ) is smaller than $0.07 \mathrm{MHz}$, equivalent to a standard deviation in speed of $\sim 4.4 \mathrm{~cm} / \mathrm{s}$.

An error bias can be appreciated when comparing the mean of the measured Doppler shifts $(21.4 \mathrm{MHz}$ is a representative value) with the expected value $(18.4 \mathrm{MHz})$ corresponding to the target rim speed. We attribute this error to an undesired angle variation of about $5.5 \mathrm{deg}$.

Figure 7 presents analogous measurements for the target displaced $80 \mathrm{~cm}$ farther than the nominal distance. As we
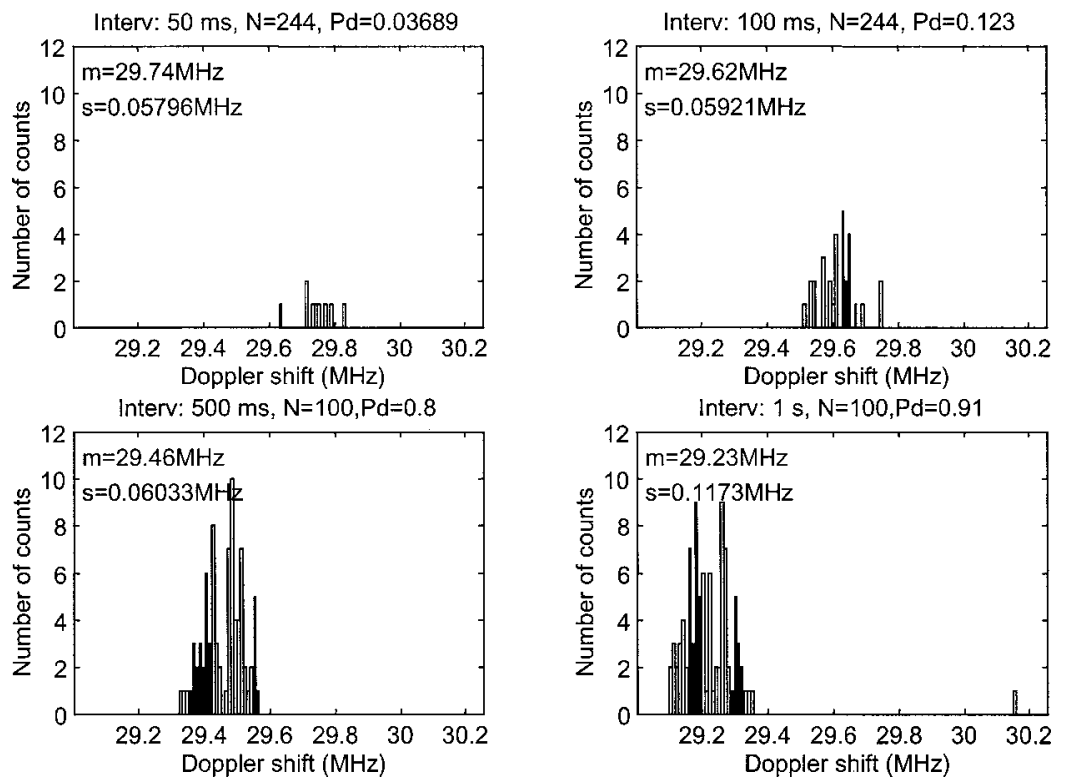

Fig. 11 Histograms of individual counts with the disk target at nominal distance 6 m plus $40 \mathrm{~cm}$ of tolerance displacement, incidence angle of $45 \mathrm{deg}$, and rotating at constant speed $444 \mathrm{rpm}$. 

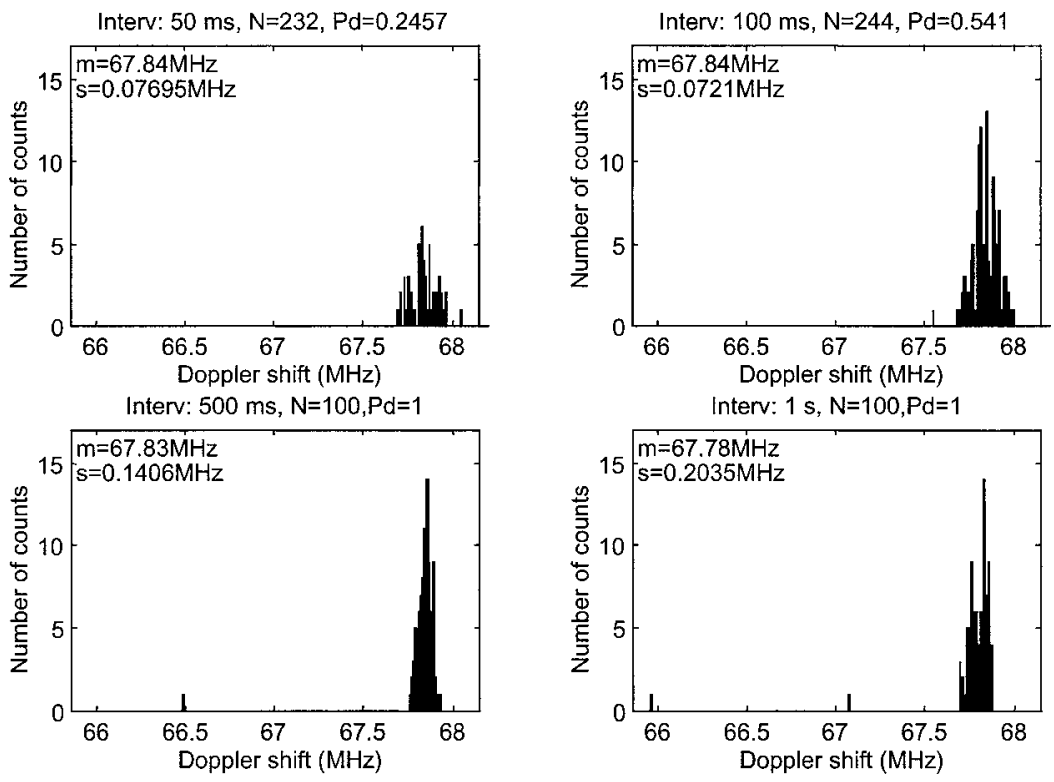

Fig. 12 Histograms of individual counts with the disk target at nominal distance $6 \mathrm{~m}$, incidence angle of $45 \mathrm{deg}$, and rotating at constant speed $1260 \mathrm{rpm}$.

can see, this fact provokes a reduction of the estimated probability of detection that is noticeable mainly in the short-interval histograms. According to this result, the system is fully operative even when the target is displaced from the nominal position, but only with long observation intervals. Another effect is the degradation of the standard deviation: for a 500-ms interval it is $0.19 \mathrm{MHz}$ (equivalent to a speed $\sim 12 \mathrm{~cm} / \mathrm{s}$ ), due to some spurious measurements. A systematic error in the determination of target rim speed is detected, similar to that of Fig. 6.

Figures 8 and 9 present the measurements taken when the rotating speed of the target is increased to $1260 \mathrm{rpm}$, equivalent to a rim linear speed $\sim 33 \mathrm{~m} / \mathrm{s}$. The theoretical and practical results included in Ref. 6 concluded that the Doppler signal peak power detected should decrease when the rotating speed increases. Comparing Figs. 6 and 8 (both with the target at the nominal distance), no worsening in probability of detection can be appreciated; this is probably because the reduction in detected signal power is not enough to reduce $P_{D}$. On the other hand, comparing Figs. 7 and 9 , a severe reduction of $P_{D}$ can be observed, because of the combination of the two effects (target displaced from its nominal position and increased rotating speed); we could not even obtain one individual count for the 50-ms
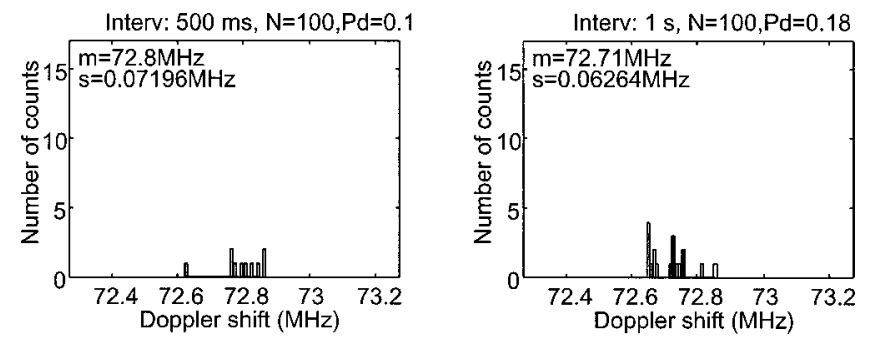

Fig. 13 Histograms of individual counts with the disk target at nominal distance $6 \mathrm{~m}$ plus $40 \mathrm{~cm}$ of tolerance displacement, incidence angle of $45 \mathrm{deg}$, and rotating at constant speed $1260 \mathrm{rpm}$. interval. The standard deviation remains under $0.1 \mathrm{MHz}$ (equivalent to $\sim 6.3 \mathrm{~cm} / \mathrm{s}$ for this incidence angle value) for all cases. A better accuracy is found in these two figures (the expected Doppler shift value is $52 \mathrm{MHz}$ ), due to a smaller error in the determination of the incidence angle.

Figures $10-13$ present analogous results when incidence angle is $\varphi=45 \mathrm{deg}$. For this incidence angle, the maximum theoretical tolerance in distance is $40 \mathrm{~cm}$, as stated in Figs. 11 and 13. According to Ref. 6 an extra reduction of detected Doppler signal power is expected, mainly due to the reduction in the backscattering; nevertheless, the probability of detection figures remain quite acceptable, except for the Fig. 13, where the combination of relatively high speed and target out of nominal position results in a nonoperative situation. In the worst case, a standard deviation value of

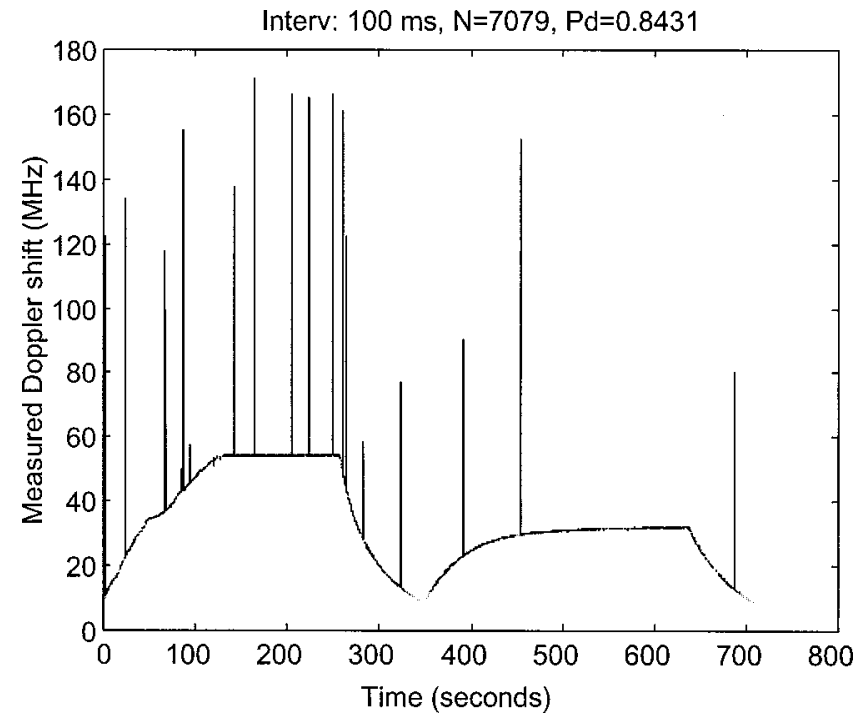

Fig. 14 Sequence of individual counts obtained with the disk target speed varying. 

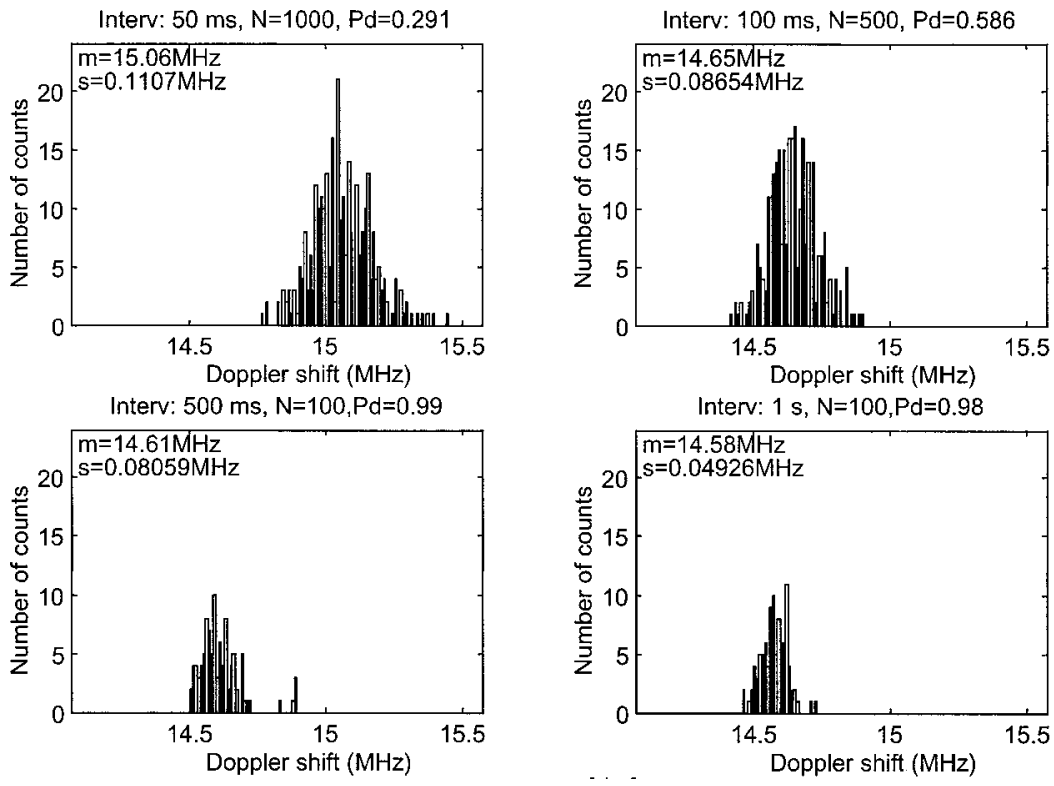

Fig. 15 Histograms of individual counts with the belt target at nominal distance $1 \mathrm{~m}$, incidence angle of $60 \mathrm{deg}$, and belt constant speed $5 \mathrm{~m} / \mathrm{s}$.

$\sim 0.2 \mathrm{MHz}$ (equivalent to $\sim 9 \mathrm{~cm} / \mathrm{s}$ for this incidence angle value) still can be guaranteed for all cases. The expected value of Doppler shift for Figs. 10 and 11 is $26 \mathrm{MHz}$, and for Figs. 12 and 13 it is $73.7 \mathrm{MHz}$, so that pointing errors around \pm 5 deg can be inferred.

\subsubsection{Measurements at variable speed}

Figure 14 presents the measurements taken from the rotating speed when it changes its velocity. This target shows a high value of inertia momentum, making impossible any abrupt change in velocity. The results can be seen in Fig. 14 (including some out of range measurements due to wrong detections). Note that the distance and angle conditions are better than those of the previous section, resulting in a probability of detection of $84 \%$ for an observation interval of $100 \mathrm{~ms}$.

\subsection{Measurements with a Belt Target}

The belt of the belt target presents a vibration that provokes high dispersion of the detected frequency of the Doppler signal, when observed in a spectrum analyzer. In Ref. 8, dispersion values over $2.5 \mathrm{MHz}$ (equivalent to a velocity $\sim 1 \mathrm{~m} / \mathrm{s}$ ) are reported. This fact, that can be easily observed when performing measurements of moving cables, for example, makes the carrier seeker subsystem especially useful. The reduced value of the scattering coefficient of the
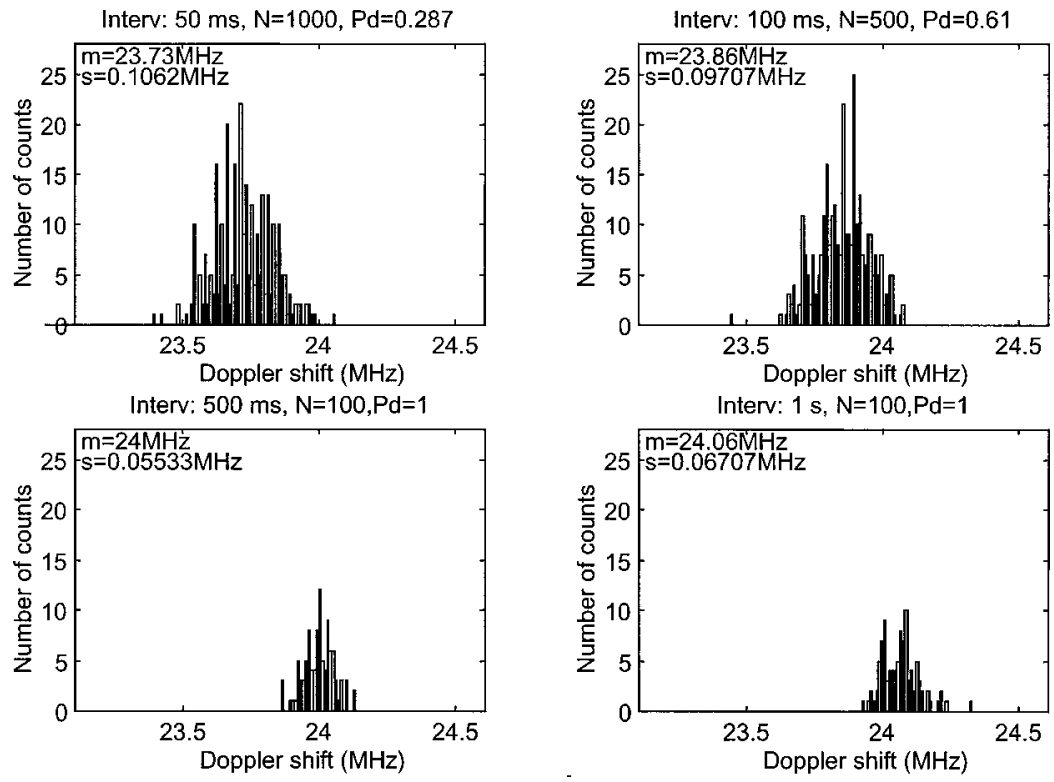

Fig. 16 Histograms of individual counts with the belt target at nominal distance $1 \mathrm{~m}$, incidence angle of $60 \mathrm{deg}$, and belt constant speed $8.2 \mathrm{~m} / \mathrm{s}$. 

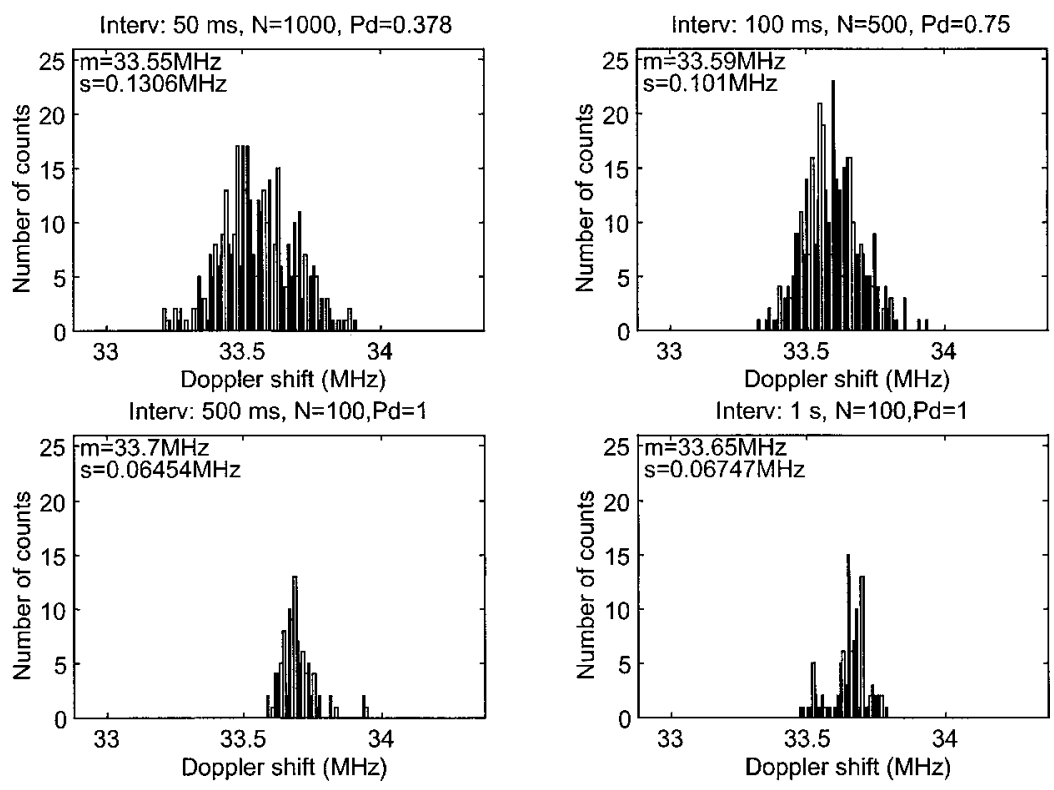

Fig. 17 Histograms of individual counts with the belt target at nominal distance $1 \mathrm{~m}$, incidence angle of $60 \mathrm{deg}$, and belt constant speed $11.5 \mathrm{~m} / \mathrm{s}$.

belt surface means that the measuring distances cannot exceed $1 \mathrm{~m}$; the reduced value of the pulley diameters makes it necessary to increase the incidence angle to $60 \mathrm{deg}$ to produce Doppler shifts over the minimum value of 10 $\mathrm{MHz}$.

\subsubsection{Measurements at constant speed}

Figures 15, 16, and 17 show the measurements taken for belt linear speed values $5,8.2$, and $11.5 \mathrm{~m} / \mathrm{s}$, respectively. Apart from the good values of the probability of detection, note that the standard deviation of the measurements is dramatically improved to values under $0.2 \mathrm{MHz}(\sim 7.3 \mathrm{~cm} / \mathrm{s})$, similar to those of the disk target. Systematic errors in Dop-

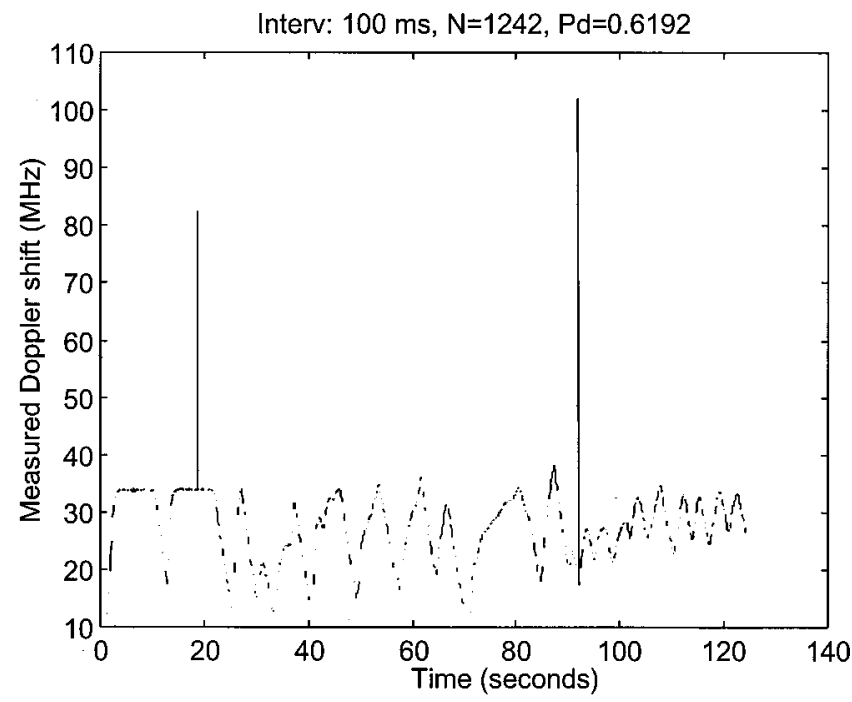

Fig. 18 Sequence of individual counts obtained with the belt target speed varying. pler shift detection, similar to those of the measurements made on the disk target, were detected; we also attribute these errors to pointing inaccuracy.

\subsubsection{Measurements at variable speed}

The belt target responds very rapidly to power supply voltage changes, so that quick speed variation can be obtained. Figure 18 shows the Doppler shift values detected. The dotted aspect of the trace is due to count failing periods, which results in a probability of detection as low as $62 \%$. Nevertheless, the speed evolution curve can be easily interpolated.

\section{Conclusions}

The performance assessment of a homodyne laser radar prototype for surface displacement monitoring was presented. The prototype uses a conditioning, acquisition, and frequency counting subsystem (carrier seeker), based on the spectrum analyzer principle, to overcome the high noise level of the Doppler signal.

Measurements presented include constant and velocity measurements of both a disk and a belt target. The probability of detection estimate has good values for the high values of the observation interval $(\geqslant 500 \mathrm{~ms})$ while at short intervals (50 and $100 \mathrm{~ms}$ ) this performance figure is very dependent on distance to target or position tolerance. The standard deviation in velocity estimation is better than 10 $\mathrm{cm} / \mathrm{s}$.

Nevertheless, some unavoidable pointing errors made it impossible to assess the accuracy of the system.

\section{Acknowledgments}

The Spanish government supported this work through programs CICYT TIC 431-93 and AMB96-1144-C02-02. 


\section{References}

1. M. J. Rudd, "A laser Doppler velocimeter employing the laser as a mixer-oscillator," J. Phys. E I(Ser. 2), 723-726 (1968).

2. J. H. Churnside, "Laser Doppler velocimetry by modulating a $\mathrm{CO}_{2}$ laser with backscattered light," Appl. Opt. 23(1), 61-66 (1984).

3. J. H. Churnside, "Signal-to-noise in a backscattered-modulated Doppler velocimeter," Appl. Opt. 23(13), 2097-2106 (1984).

4. D. H. Liskow and J. N. Paranto, "Optimization of laser beam spread to maximize laser radar probability of detection," in Laser Radar IV, Proc. SPIE 1103, 83-91 (1989).

5. P. Gatt, S. W. Henderson, J. A. L. Thompson, and D. L. Bruns, "Micro-Doppler lidar signals and noise mechanisms: theory and experiment," in Laser Radar Technology and Applications V, Proc. SPIE 4035, 422-435 (2000).

6. A. Rodriguez, A. Comeron, and D. Garcia, "Homodyne laser radar system for surface displacement monitoring," Opt. Eng. 40(3), 398405 (2001)

7. I. C. Potter, "Frequency response of the 6328- $\AA$ helium-neon laser interferometer," J. Appl. Phys. 40(12), 4770-4776 (1969).

8. A. Rodriguez, "Sistemas lidar coherentes e incoherentes de baja potencia para la medida de blancos solidos," PhD Dissertation, Universitat Politécnica de Catalunya (1998).

9. A. Papoulis, Probability, Random Variables and Stochastic Processes, 2nd ed., McGraw-Hill, New York (1984).

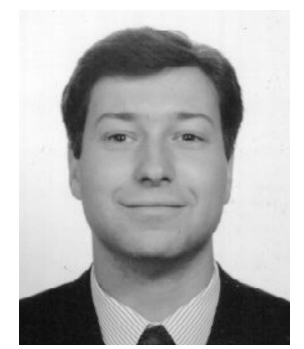

systems.
Alejandro Rodriguez received the telecommunication engineer degree from the Technical University of Madrid (UPM) in 1993 and the $\mathrm{PhD}$ in telecommunication engineering from the Technical University of Catalonia (UPC) in 1998. Since 1995, he has been an associate professor with the Department of Signal Theory and Communications (UPC). His research interests include low power coherent and incoherent laser radar systems and atmospheric lidar

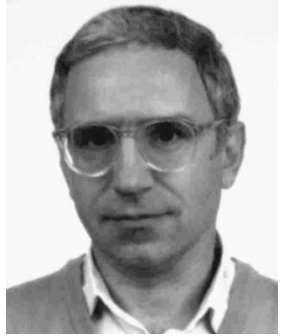

Adolfo Comeron received a telecommunication engineer degree from the Telecommunication Engineer School of Barcelona in 1976 and DEA and Drlng degrees from the Paris-XI University, Orsay, in 1977 and 1980 , respectively. He is currently a professor at the Technical University of Catalonia, Barcelona, Spain. His research activities have included the study of nonlinear devices at IR wavelengths and the development of microwave and millimeter-wave receivers for satellite communication systems. They currently focus on free-space optical communications and remote detection and sensing at optical wavelengths. He is a member of SPIE.

Eloy Mesalles recived a telecommunication engineer degree from the Telecommunication Engineer School of Barcelona in 1998. He is currently with NORTEL HISPANIA. 